\title{
On the ergoregion instability in rotating gravastars
}

\author{
Cecilia B. M. H. Chirenti ${ }^{1}$ and Luciano Rezzolla $a^{1,2,3}$ \\ ${ }^{1}$ Max-Planck-Institut für Gravitationsphysik, Albert Einstein Institut, 14476 Golm, Germany \\ ${ }^{2}$ Department of Physics, Louisiana State University, Baton Rouge, LA 70803 USA \\ ${ }^{3}$ INFN, Department of Physics, University of Trieste, Trieste, Italy
}

(Dated: October 24, 2018)

\begin{abstract}
The ergoregion instability is known to affect very compact objects that rotate very rapidly and do not possess a horizon. We present here a detailed analysis on the relevance of the ergoregion instability for the viability of gravastars. Expanding on some recent results, we show that not all rotating gravastars are unstable. Rather, stable models can be constructed also with $J / M^{2} \sim 1$, where $J$ and $M$ are the angular momentum and mass of the gravastar, respectively. The genesis of gravastars is still highly speculative and fundamentally unclear if not dubious. Yet, their existence cannot be ruled out by invoking the ergoregion instability. For the same reason, not all ultra-compact astrophysical objects rotating with $J / M^{2} \lesssim 1$ are to be considered necessarily black holes.
\end{abstract}

PACS numbers: 04.40.Dg, 04.30.Nk, 04.25.Nx

\section{INTRODUCTION}

Gravastars have been recently presented by Mazur and Mottola [1] as a new exact solution to the Einstein equations. In the original suggestion these are compact, spherically symmetric and non-singular objects, that can be taken to be almost as compact as black holes. In this "three-layer" model, the high compactness is supported by a de-Sitter core, surrounded by a shell of matter, and the exterior vacuum spacetime is, of course, that of the Schwarzschild solution.

According to the original picture proposed in ref. [1], a massive collapsing star could go through a phase transition when its radius approaches $R=2 M$, forming a gravastar instead of a black hole. Although the dynamical processes that would lead to the formation of a gravastar are far from being understood and could probably be described as rather exotic, the final state can be described by an exact (and fairly simple) solution of the Einstein equations. This is what stimulates our interest in this matter.

The original gravastar model has inspired many subsequent works, but always describing stationary solutions (except from ref. [2], as we will see below). A related simplified model with an infinitesimally thin shell was proposed in ref. [3] and later also generalized in ref. [4]. Several possibilities for the interior solution have been considered in refs. [5], [6] and [7], among others. More recently, a solution for electrically charged gravastar configurations was proposed in ref. [8] and limits on the existence of gravastars from astronomical data were considered in ref. [9].

Also an effort has been made to assess the properties of gravastars when these are perturbed [10,11]. The result of this analysis has lead, among other things, to the evidence that perturbations in spherical gravastars can be used to discriminate them from black holes, thus removing one of the most serious consequences of the existence of such ultra-compact objects [11]. Note that hereafter we define as ultra-compact any stellar object whose compactness $\mu \equiv M / R$ is much larger than that of typical neutron stars of comparable mass and angular momentum, and which is $\mu \sim 0.15-0.2$. This definition is inevitably weak and ambiguous, but it aims at focussing on the large compactness of gravastars as the most relevant property. Clearly, black holes are always more compact than any possible gravastar model (although their compactness is only infinitesimally smaller for nonrotating models) but the relevant point to bear in mind is that gravastars have compactness much larger than that of standard stars and comparable (although smaller) to that of black holes.

Further expanding on the perturbative analysis carried out in ref. [11], Cardoso et al. [2] have recently considered the properties of perturbed and rotating gravastars and assessed, within the slow-rotation approximation, their stability against the "ergoregion instability". We recall that such instability affects rapidly rotating and very compact stellar objects which do have an ergoregion but do not have an event horizon [12, 13].

The Kerr black hole is a good example of an object that has an ergoregion. But an ergoregion can also develop in compact stars that are sufficiently rapidly rotating. In this region, the relativistic frame dragging is so strong that no stationary orbits are allowed. All trajectories of particles in this region must rotate in the same direction of the rotation of the star.

Because of this effect, some particles in the ergoregion can be measured by an observer at rest at infinity as having negative energy. This happens because this observer measures the energy of the particles by projecting their four-momentum vectors onto his four-velocity. As the observer is stationary (and no stationary trajectories are allowed inside the ergoregion), his four-velocity is outside of the light cone of the particles in the ergoregion. This causes some of these particles to have their energy measured as negative by the observer at infinity.

The instability occurs then in the following way. From an initially small perturbation with negative energy trapped in the ergoregion, one can extract positive energy (that leaves the star and goes to infinity) by increasing the negative energy inside the ergoregion (thus conserving the total energy). As the negative energy trapped in the ergoregion increases, the star radiates even more positive energy to infinity and this process leads to the instability. This process is very general, and scalar, electromagnetic and gravitational waves become unstable in a star with an ergoregion. 
This paper is dedicated to reconsider the analysis carried out by Cardoso et al. [2] and to extend it to a larger space of possible models, taking into account the limits on the thickness of the matter shell and on its compactness. When doing this, we confirm the results of Cardoso et al. [2] for their models, but also show that the conclusions drawn were excessively restrictive. In particular we show that not all rotating gravastars are unstable to the ergoregion instability. Rather, we find that models of rotating gravastars without an ergoregion (and therefore stable) can be constructed also for extreme rotation rates, namely for models with $J / M^{2} \geq 1$, where $J$ and $M$ are the gravastar's angular momentum and mass, respectively.

The paper is organized as follows: in Section $\amalg$ we briefly review our gravastar model and the slow-rotation approximation. In Section $\amalg$ we develop the equations for scalar perturbations and present the WKB approximation used. In Section IV we present our results and analyze the behavior of the instability in the space of parameters and in Section $\nabla$ we present our concluding remarks. We use $c=G=1$ throughout the paper.

\section{A ROTATING GRAVASTAR MODEL}

We start our analysis by adding a uniform rotation to the simple fluid gravastar model with anisotropic pressure presented in ref. [11]. We recall that the use of anisotropic pressures was introduced by Cattoen et al. [14] to remove in part the complications produced by the infinitesimal shells in the original gravastar model of Mazur and Mottola [1]. In this way, the anisotropic pressure replaces the surface tension introduced by the matching of the metric in the infinitesimally thin shells. Although the use of an anisotropic pressure is essentially arbitrary, as arbitrary are the equations of state used to describe such a pressure, it has the appealing property of being continuous and thus of allowing one to build equilibrium models without the presence of infinitesimally thin shells and thus look more seriously into the issue of stability.

We write therefore the line element for a finite-thickness rotating gravastar with anisotropic pressures within the slowrotation approximation, (namely at first-order in the angular velocity in the small parameter $\Omega / \Omega_{K}$, where $\Omega_{K}$ is the Keplerian limit) as

$$
\begin{array}{r}
d s^{2}=-e^{\nu(r)} d t^{2}+e^{\lambda(r)} d r^{2}+r^{2} d \theta^{2}+ \\
+r^{2} \sin ^{2} \theta(d \phi-\omega(r) d t)^{2}
\end{array}
$$

together with the energy momentum tensor given by

$$
T_{\nu}^{\mu}=\left(\rho+p_{\mathrm{t}}\right) u^{\mu} u_{\nu}+p_{\mathrm{t}} \delta_{\nu}^{\mu}+\left(p_{\mathrm{r}}-p_{\mathrm{t}}\right) s^{\mu} s_{\nu}
$$

where $\rho$ is the energy density of the gravastar, $p_{\mathrm{r}}$ and $p_{\mathrm{t}}$ are the radial and tangential pressures, respectively. The vector $u_{\mu}$ is the fluid four-velocity,

$$
\begin{gathered}
u^{\mu} u_{\mu}=-1, \quad u^{r}=u^{\theta}=0, \quad u^{\phi}=\Omega u^{t}, \\
u^{t}=\left[-\left(g_{t t}+2 \Omega g_{t \phi}+\Omega^{2} g_{\phi \phi}\right)\right]^{-1 / 2},
\end{gathered}
$$

and the vector $s^{\mu}$ is orthogonal to the fluid four-velocity,

$$
s^{\mu} s_{\mu}=1, \quad u^{\mu} s_{\mu}=0 \quad\left(s_{t}=s_{\phi}=0\right) .
$$

The functions $\nu(r)$ and $\lambda(r)$ in the line element (1) are given in terms of the mass $m(r)$ and the radial pressure $p_{r}(r)$, as in the non-rotating case,

$$
\begin{array}{r}
e^{-\lambda}=1-\frac{2 m(r)}{r}, \\
\nu^{\prime}=\frac{2 m(r)+8 \pi r^{3} p_{r}}{r(r-2 m(r))},
\end{array}
$$

where a prime denotes a (total) radial derivative. As usual, we define the gravitational mass within a radius $r$ as $m(r) \equiv$ $\int_{0}^{r} 4 \pi r^{2} \rho d r$ and

$$
\rho(r)= \begin{cases}\rho_{0}, & 0 \leq r \leq r_{1} \\ a r^{3}+b r^{2}+c r+d, & r_{1}<r<r_{2} \\ 0, & r_{2} \leq r\end{cases}
$$

with the coefficients $a, b, c, d$ given by

$$
\begin{array}{r}
a=\frac{2 \rho_{0}}{\delta^{3}}, \\
b=-\frac{3 \rho_{0}\left(r_{2}+r_{1}\right)}{\delta^{3}}, \\
c=\frac{6 \rho_{0} r_{1} r_{2}}{\delta^{3}}, \\
d=\frac{\rho_{0}\left(r_{2}^{3}-3 r_{1} r_{2}^{2}\right)}{\delta^{3}},
\end{array}
$$

where $\delta \equiv r_{2}-r_{1}$ is the "thickness" of the gravastar and

$$
\rho_{0}=\frac{15 M}{2 \pi\left(r_{1}+r_{2}\right)\left(2 r_{1}^{2}+r_{1} r_{2}+2 r_{2}^{2}\right)} .
$$

The equation of state used for $p_{r}(\rho)$ serves here only as a closure relation and is therefore chosen to be in the simplest possible form, namely a polynomial of the type [10, 15]

$$
p_{r}(\rho)=\left[\alpha-(\alpha+1)\left(\frac{\rho}{\rho_{0}}\right)^{2}\right]\left(\frac{\rho}{\rho_{0}}\right) \rho,
$$

where $\alpha=2.2135$ is determined by demanding that the maximum sound speed $c_{\mathrm{s}}^{2}$ at which $d^{2} p_{r} / d \rho^{2}=0$ coincides with the speed of light to rule out a superluminal behavior. Finally, the tangential pressure $p_{t}$ is given by the anisotropic TOV equation

$$
p_{t}=p_{r}+\frac{r}{2} p_{r}^{\prime}+\frac{1}{2}\left(p_{r}+\rho\right)\left[\frac{m(r)+4 \pi r^{3} p_{r}}{r(1-2 m(r) / r)}\right] .
$$

The function $\omega(r)$ can be shown to be of first order in the angular velocity $\Omega$ and it describes the dragging of inertial frames. Formally, it can be obtained from the ${ }_{\phi}^{t}$ component of the field equations [16]

$$
R_{\phi}^{t}=8 \pi T_{\phi}^{t}
$$


which gives, for our anisotropic case,

$$
\varpi^{\prime \prime}+\left(\frac{4}{r}-\frac{4 \pi r^{2}\left(\rho+p_{r}\right)}{r-2 m}\right) \varpi^{\prime}=\frac{16 \pi r\left(\rho+p_{t}\right)}{r-2 m} \varpi
$$

where

$$
\varpi(r)=\Omega-\omega(r),
$$

for the region of the spacetime interior to the gravastar. It is easy to see that eq. (15) is equivalent to eq. (2.25) of ref. [2]. In the exterior region, on the other hand, we have

$$
\varpi(r)=\Omega-\frac{2 J}{r^{3}}, \quad \text { or } \quad \omega(r)=\frac{2 J}{r^{3}} .
$$

The definition of $\varpi$ in eq. (16) deserves some attention and it corresponds to the difference between $\Omega$, the angular velocity of the gravastar (as seen by an observer at rest), and $\omega(r)$, which gives the angular velocity of a zero angular momentum observer (ZAMO). Therefore, $\varpi(r)$ is the angular velocity of the gravastar as seen by the ZAMO.

As a general result in the asymptotically flat limit, the dragging must go to zero as $\omega=2 J / r^{3}+O\left(r^{-4}\right)$ for $r \rightarrow \infty$, thus defining $J$ as the angular momentum of the spacetime [17]. Demanding now that the exterior equations (17) to be consistent with this asymptotic limit implies immediately that the integration constant $J$ in (17) should be identified as the total angular momentum of the gravastar.

A representative behavior of the frame-dragging function $\omega(r)$ is shown in Fig. 11 for a gravastar with thickness of the shell $\delta / M=0.4$ and compactness $\mu \equiv M / r_{2}=0.45$. The frame-dragging is obtained by numerically integrating eq. 15, with initial conditions $\varpi^{\prime}(r=0)=0$ and $\varpi(r=0)$ finite. As shown in the figure, the solution for $\omega(r)$ in the interior of the gravastar is constant [cf. eqs. (15) and (16)] and monotonically decreases outwards. The two integration constants $\Omega$ and $J$ can then be determined by matching the interior and exterior solutions at the boundary $r=r_{2}$. Finally, in the exterior of the gravastar, $\omega(r)$ goes asymptotically to zero [cf. eq. [17]].

\section{SCALAR PERTURBATIONS AND THE ERGOREGION INSTABILITY}

We recall that if a compact relativistic star rotates sufficiently fast, it will posses an ergoregion, namely a region in which the frame-dragging is so intense that stationary orbits are no longer possible. Within this region even massless particles, such as photons, will be involved in the "drag" and all trajectories will rotate in the prograde direction. Mathematically, the boundary of the ergoregion is defined as where the covariant $t t$-component of the metric has a zero, i.e.

$$
g_{t t}=-e^{\nu}+r^{2} \omega^{2} \sin ^{2} \theta=0,
$$

and it should be noted that the ergoregion does not need to be restricted to the exterior of the compact star and, rather, it can also involve regions interior to the star.

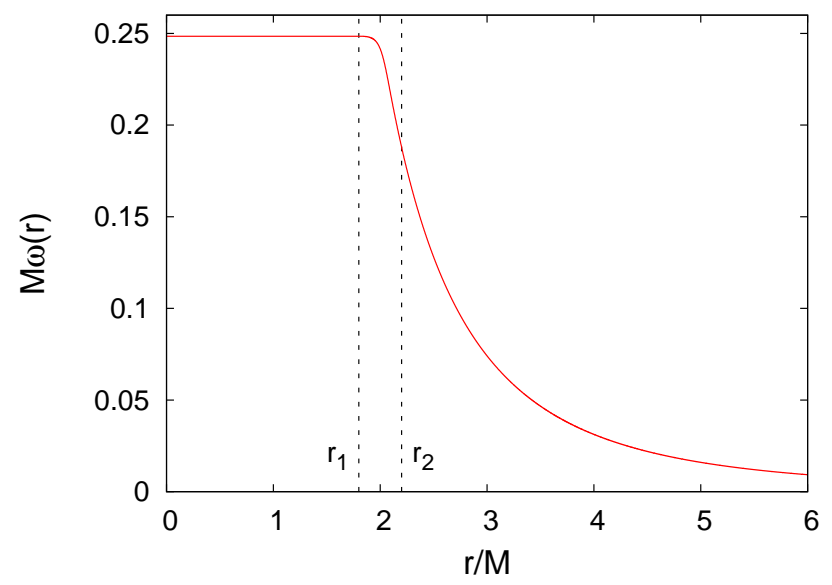

FIG. 1: Typical example for the dragging of inertial frames $\omega(r)$, for a gravastar with $\mu=0.45, \delta / M=0.4$ and $J / M^{2}=1\left(\Omega / \Omega_{K}=\right.$ $0.82)$. We can see that $\omega=$ const. in the interior $\left(r<r_{1}\right)$ and $\omega \rightarrow 0$ in the exterior region $\left(r>r_{2}\right)$.

The main interest in the existence of an ergoregion in a compact star stems from the fact that these regions lead to a secular instability by means of which any initially small perturbation will grow exponentially in time [2, 12]. Clearly, one expects that nonlinear effects will intervene to limit the growth of the instability once this has reached a sufficiently high (saturation) amplitude which, however, cannot be determined on the basis of a linear perturbative investigation. While determining such an amplitude is of great physical interest, we will here limit ourselves to a simple linear analysis of the problem. On the other hand, we will determine not only if an instability can or cannot develop but also, and more importantly, the characteristic timescale for the growth of the instability. Indeed, as shown already many years ago in ref. [12], for ultracompact uniform-density stars, the timescale for the growth of scalar perturbations via the ergoregion instability can sometimes be several orders of magnitude larger than the age of the universe. When this is case, the instability grows so slowly that the stars are effectively stable even if the linear analysis reveals that they are mathematically unstable.

Hereafter we will concentrate on scalar perturbations and within the WKB approximation. There are many references in the literature where this method is described, but we suggest for instance ref. [18]. The WKB method (from Wentzel, Kramers, Brillouin) is used to approximate the solution of differential equations of the general form

$$
y^{\prime \prime}(x)+\omega^{2} r(x) y(x)=0,
$$

where $\omega^{2} \gg 1$. As we will see below, the scalar perturbations that we are considering are described by eq. 222), which has the same form as eq. (19) above. The same equation is often found to arise in quantum mechanics, but also in classical physics problems. The key point of the approximation is the assumption $\omega^{2} \gg 1$ (high frequency approximation in quantum mechanics). It allows us to consider that, for every 
$\omega_{n} \gg 1$, then $r(x)$ is approximately constant between two zeros of $y_{n}(x)$. It is easy to see that this approximation greatly simplifies the integration of eq. (19).

Of course, studying the response of a rotating gravastar to electromagnetic or gravitational perturbations would be astrophysically more interesting and realistic. However, the use of scalar perturbations has the important advantage that in this case one can decompose the perturbations in spherical harmonics and reduce the perturbation equation to a single ordinary differential equation, which can be integrated with very modest computational costs and high accuracy. In addition, because the order of magnitude for the growth of the perturbations is expected to be the same for scalar, electromagnetic and gravitational perturbations [this is indeed the case for the decay of perturbations of different spins in a Schwarzschild spacetime (see ref. [19] for some numerical results)], estimating the growth time of scalar perturbations allows for a simple and direct extension also to other types of perturbations.

Bearing this in mind, we next proceed to the study of the massless scalar wave equation in the slowly rotating gravastar background that we introduced in Section $\amalg$ We briefly review here the basic steps usually followed in this type of analysis; such steps can be found in many papers and are reproduced here only for completeness. The wave equation

$$
\frac{1}{\sqrt{-g}} \frac{\partial}{\partial x^{\mu}}\left(\sqrt{-g} g^{\mu \nu} \frac{\partial \psi}{\partial x^{\nu}}\right)=0,
$$

can be separated by using the ansatz

$$
\begin{aligned}
\psi(t, r, \theta, \phi) & =\bar{\chi}(r) \exp \left\{-\frac{1}{2} \int\left(\frac{2}{r}+\frac{\nu^{\prime}}{2}-\frac{\lambda^{\prime}}{2}\right) d r\right\} \times \\
& \times e^{\mathrm{i} \sigma t} Y_{\ell m}(\theta, \phi) .
\end{aligned}
$$

where the eigenfrequency $\sigma$ is in general complex, with $\sigma=$ $\sigma_{r}-i / \tau$. As a result, $\tau>0$ leads to an exponential growth, while $\tau<0$ leads to a decay.

In the high $m$ limit (and taking $\ell=m$ ), eq. (20) can be written using eq. 21) and keeping only the dominant terms (i.e. first order in $\omega$ and $1 / m$ ) as

$$
\bar{\chi}_{, r r}+m^{2} T(r, \Sigma) \bar{\chi}=0,
$$

where $m$ is here the order of the $Y_{\ell m}(\theta, \phi)$ spherical harmonics (not to be confused with the mass function $m(r)$ ), $\Sigma=\sigma / m$ is the negative of the pattern speed of the perturbation and $T$ is given in terms of the two rotationally split "effective potentials" $V_{+}$and $V_{-}$,

$$
\begin{aligned}
T & \equiv e^{\lambda-\nu}\left(\Sigma-V_{+}\right)\left(\Sigma-V_{-}\right), \\
V_{ \pm} & \equiv-\omega \pm \frac{e^{\nu / 2}}{r} .
\end{aligned}
$$

It is easy to see that the boundary of the ergoregion given in eq. (18) coincides, in the equatorial plane, with $V_{+}=0$, so that the ergoregion is effectively contained in the region where $V_{+}<0$.

Our stability analysis translates therefore to finding the complex eigenfrequencies $\sigma$ of the scalar wave modes that

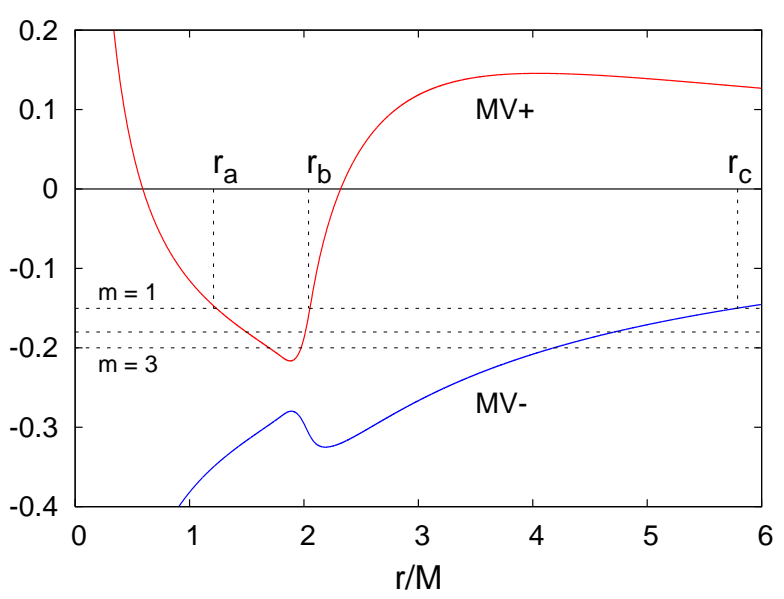

FIG. 2: Typical example for the potentials $V_{ \pm}$, for a gravastar with $\mu=0.45, \delta / M=0.4$ and $J / M^{2}=1\left(\Omega / \Omega_{K}=0.82\right)$. The first three unstable modes with negative energy "trapped" in the potential well are depicted, as well as the points $r_{a}, r_{b}$ and $r_{c}$ for the $\ell=m=$ 1 mode.

satisfy eq. 22). However, because these frequencies generally have the real part being much larger than the imaginary one, i.e. , $\Re(\sigma) \gg \Im(\sigma)$ (or, equivalently, $\sigma_{r} \gg 1 / \tau$ ), it is a reasonable approximation to consider $\sigma$ as essentially a real number. It is also more convenient to the problem to use $\Sigma=\sigma / m$ instead of $\sigma$ itself (see the Appendix for more details).

Furthermore, when considered within the WKB approximation, the potentials $V_{ \pm}(r)$ determine four different regions which are reminiscent of those appearing for waves trapped in the potential well of $V_{+}$. To illustrate this in more detail we show in Fig. 2 an example of the two potentials for some typical parameters. In addition, the figure shows with dotted horizontal lines the frequencies of the first three unstable modes, i.e. $\Sigma_{m=1}, \Sigma_{m=3}$, so that $\Sigma^{2}$ can be viewed as an analog of the energy of a quantum mechanical particle. For each of the unstable modes there is therefore an inner "forbidden" region $0<r<r_{a}$, an "allowed" region $r_{a}<r<r_{b}$, a "potential barrier" $r_{b}<r<r_{c}$ and an external "allowed" region $r>r_{c}$. The points $r_{a}, r_{b}$ and $r_{c}$ shown in the figure correspond to the $\ell=m=1$ mode.

The WKB matching of the wave functions in the four regions is shown in the Appendix, where we present the derivation of eqs. 25 and (26) below. The unstable modes are determined by requiring that

$$
m \int_{r_{a}}^{r_{b}} \sqrt{T} d r=\left(n+\frac{1}{2}\right) \pi ; \quad n=0,1,2, \ldots
$$

and

$$
\tau=4 \exp \left(2 m \int_{r_{b}}^{r_{c}} \sqrt{|T|} d r\right) \int_{r_{a}}^{r_{b}} \frac{d}{d \Sigma} \sqrt{T} d r
$$



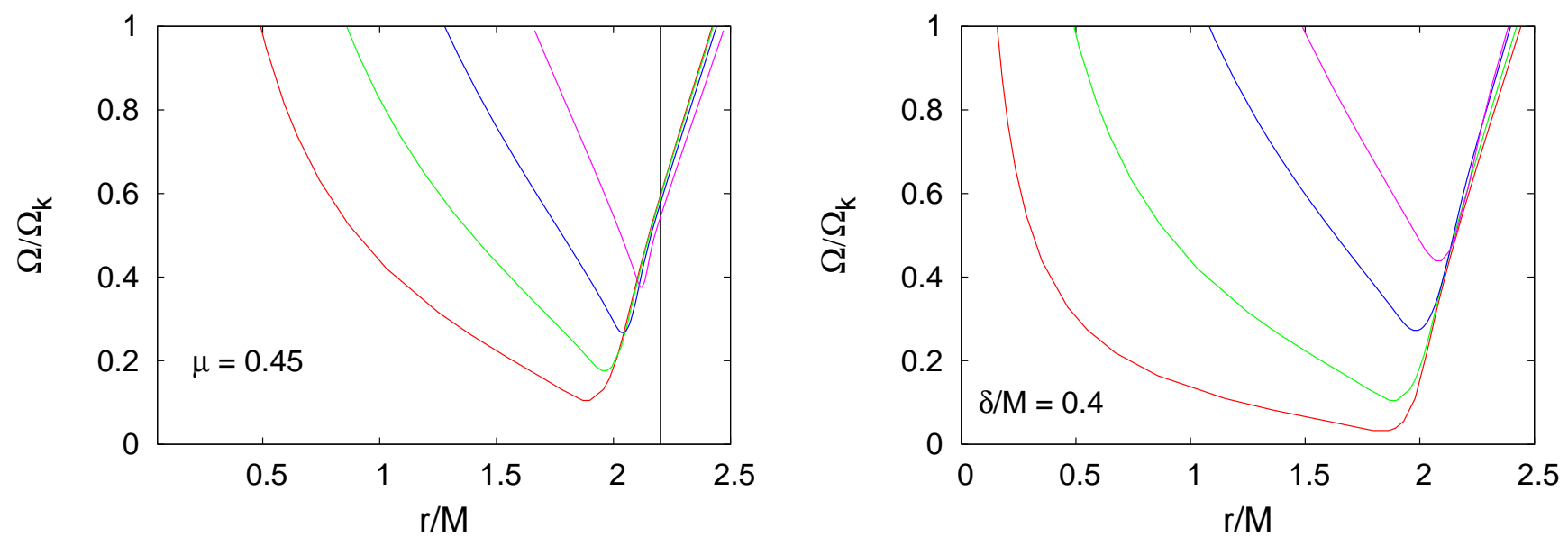

FIG. 3: Left panel: Change in the size of the ergoregion for fixed compactness $\mu=0.45$, several different values for $\delta / M$ (from top to bottom $\delta / M=0.1,0.2,0.3$ and 0.4 ) and increasing angular velocity $\Omega$, until the Keplerian limit $\Omega_{K}$. A given value of $\Omega$ on the vertical axis determines the inner and outer radii of the ergoregion, while the vertical line shows the location of the radius of the gravastar for all the models: the ergoregion generally starts in the interior of the gravastar and goes up to a radius exterior to the radius $r_{2}$ of the gravastar. Right panel: Same as the left panel, but for fixed thickness of the shell $\delta / M=0.4$ and different values for $\mu$ (from top to bottom $\mu=0.42,0.43,0.45$ and 0.47 ). In this case we do not show the surface radius, since each model has a different radius $r_{2}$.

where

$$
\frac{d}{d \Sigma} \sqrt{T}=(\Sigma+\omega) \frac{e^{\lambda-\nu}}{\sqrt{T}} .
$$

Equation 25] is the classical Bohr-Sommerfeld rule and determines $\Sigma$, while eq. 26) gives the growing time of the instability. The limits of the integration interval $r_{a}, r_{b}$ and $r_{c}$ have the physical interpretation given above for Fig. 2 and correspond, mathematically, to the turning points as given by the condition $V_{+}=\Sigma$ (or $T=0$ ) and to the beginning of the free allowed region as given by the condition $V_{-}=\Sigma$. Note also that the use of the absolute value for $T$ in the first integral of eq. (26) is due to the fact that in the interval $r_{b}<r<r_{c}$ (i.e. inside the potential barrier) $T<0$ [cf. eq. (23)].

It is customary in the evaluation of the integral in eq. (25), to use an analytical parabolic approximation for $V_{+}$(see ref. [12]). When this is done, the functions $\lambda, \nu$ and $V_{-}$are taken to be constants, with their values set to $\lambda(R), \nu(R)$ and $V_{-}(R)$, where $R$ is the radius at which $V_{+}$has its minimum. This greatly simplifies the calculations and is a very useful strategy in general. In the case of a gravastar, however, the potential well in $V_{+}$is typically very asymmetric around the minimum of the potential, so the usual analytical parabolic approximation is not appropriated in this case, for it would introduce too large errors and it has not been used in the numerical solution of eq. 26.

Collected in Table $\Pi$ are some typical numerical values obtained for the potential $V_{+}$of the gravastar with $\mu=0.45$ and $\delta / M=0.4$ and different values of $J$. With these data it is possible to confirm that both the size of the ergoregion (which extends from $r_{\min }$, the inner boundary of the ergoregion, to $r_{\max }$, the outer boundary of the ergoregion, in the notation of the table) and the depth of the potential well $\left|V_{+}(R)\right|$ increase with $J$, while $R$ remains essentially unchanged.
TABLE I: Typical values of $J / M^{2}, \Omega / \Omega_{K}, r_{\min }$ (inner boundary of the ergoregion), $r_{\max }$ (outer boundary of the ergoregion), $R$ (radius at which $V_{+}$is minimum) and $V_{+}(R)$ for the gravastar with $\mu=$ 0.45 and $\delta / M=0.4$.

\begin{tabular}{llllll}
\hline \hline$J / M^{2}$ & $\Omega / \Omega_{K}$ & $r_{\min } / M$ & $r_{\max } / M$ & $R / M$ & $M V_{+}(R)$ \\
\hline 1.2 & 0.98 & 0.500 & 2.411 & 1.8789 & -0.2661 \\
1.0 & 0.82 & 0.591 & 2.321 & 1.8797 & -0.2164 \\
0.8 & 0.65 & 0.721 & 2.231 & 1.8806 & -0.1668 \\
0.6 & 0.49 & 0.917 & 2.146 & 1.8815 & -0.1172 \\
0.4 & 0.33 & 1.225 & 2.071 & 1.8824 & -0.0068 \\
0.2 & 0.16 & 1.683 & 1.987 & 1.8834 & -0.0018 \\
\hline \hline
\end{tabular}

\section{RESULTS}

We have first checked our results against the values obtained by Cardoso et al in ref. [2] (for $\Sigma$ and $\tau$ ) for a typical model with $r_{1}=1.8, r_{2}=2.2$ and $M=1$, with very good agreement. Based on their findings for this typical model, which shows a very rapidly growing instability, they have concluded that the ergoregion instability would "rule out" the possibility of rotating gravastars to exist as alternatives to Kerr black holes. However, drawing a general conclusion from a specific example can be too restrictive. In view of this, we have decided to reconsider the issue of whether all of the rotating gravastars are unstable to the ergoregion instability and if so over what timescales. As we will show in this Section, by considering a much larger space of parameters we reach conclusions which are rather different from those of ref. [2].

We start by showing in Fig. 3how the size of the ergoregion (taken at the equatorial plane) depends on the parameters of 


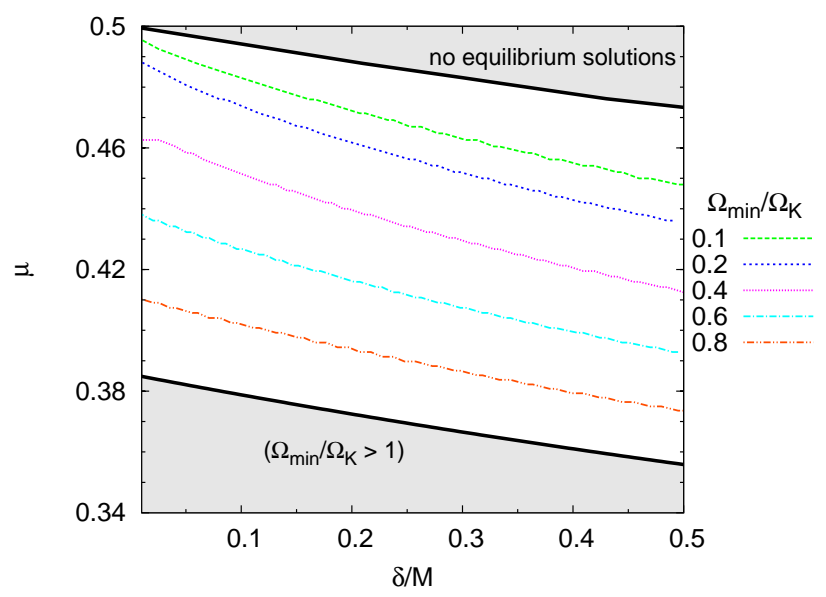

FIG. 4: Minimum angular velocity necessary for the existence of an ergoregion, as a function of both $\mu$ and $\delta$. If an ergoregion is present, the instability will set in for a high enough value of $m$. In the grey area at the bottom, an angular velocity $\Omega$ larger than the mass shedding limit $\Omega_{K}$ would be required for an ergoregion to develop. The grey area at the top shows a constraint on the possible (nonrotating) gravastar solutions found in ref. [11].

the gravastar and in particular on its compactness $\mu$, on its thickness $\delta$ and on its angular velocity $\Omega$, which we take to range from zero to the Keplerian (mass shedding) limit $\Omega_{K} \equiv$ $\left(M / r_{2}^{3}\right)^{1 / 2}$

On the left panel of Fig. 3 in particular, we report the variation of the size of the ergoregion for models with $\mu=0.45$ and different values of $\delta$, with the vertical line signing the surface of the gravastar. On the right panel, on the other hand, we obtain essentially the same behavior, but this time for models with $\delta / M=0.4$ and different values of $\mu$. These plots are to be interpreted as follows: for a given value of $\Omega / \Omega_{K}$ on the vertical axis, each of the curves in the plot provides the values of the inner and outer boundaries of the ergoregion. It is then easy to see that the ergoregion becomes obviously larger for increasing values of $\Omega$, but also for increasing values of $\delta$ and $\mu$. It also should be noted that the ergoregion is mostly (but not exclusively) contained in the interior of the gravastar, and it also extends to the exterior region. Most importantly, however, the local minima in Fig. 3 indicate the first important result of this investigation: not all rotating gravastars possess an ergoregion. Rather, for any choice of compactness and thickness of the gravastar, there exists a minimum angular velocity $\Omega_{\text {min }}$ above which an ergoregion of finite size develops. In this respect, and not surprisingly, gravastars behave like rotating compact stars. It is then a trivial consequence of the above result that not all rotating gravastars are unstable to the ergoregion instability.

This conclusion is summarized in Fig. 4, which shows the minimum angular velocity necessary for the existence of an ergoregion, as a function of both the compactness $\mu$ and thickness $\delta$ of the gravastar. In other words, for any couple of values of $(\mu, \delta)$ outside of the shaded regions, the corresponding

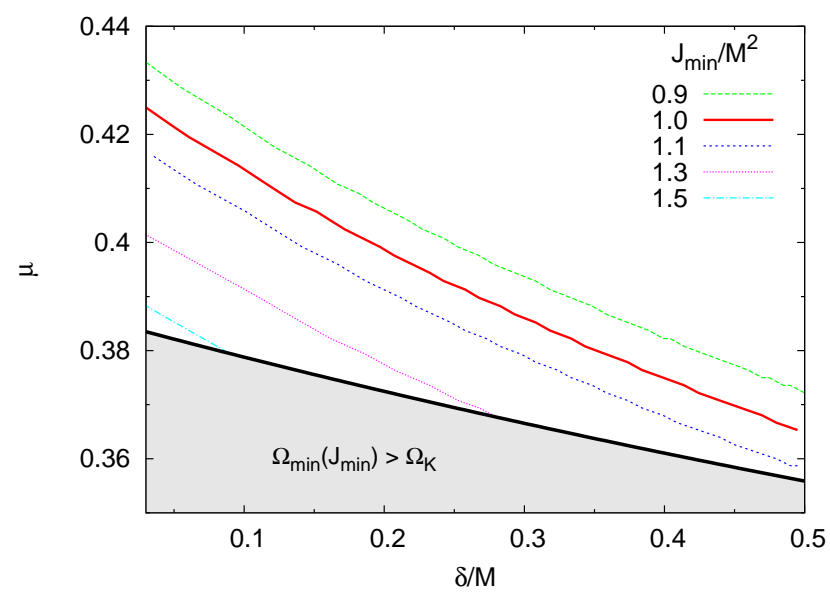

FIG. 5: Minimum angular momentum necessary for the existence of an ergoregion, as a function of both $\mu$ and $\delta$. Gravastars with parameters $(\mu, \delta)$ given below a curve labeled with some value of $J_{\min } / M^{2}$ will be stable if rotating with angular momentum smaller or equal to $J_{\min }$. Note that the $\Omega$ is not constant along the lines, and increases in the direction of the region labeled $\Omega_{\min }\left(J_{\min }\right)>\Omega_{K}$.

gravastar will not possess an ergoregion if spinning below the value of $\Omega_{\min }$ at that point. Note also that in the grey area at the bottom of the figure, an angular velocity $\Omega$ larger than the mass shedding limit $\Omega_{K}$ would be required for an ergoregion to develop. Similarly, the grey area at the top of the figure shows the constraints on the possible (nonrotating) gravastar solutions found in ref. [11]. It is important to remark that, as clearly shown in Fig. 4 , the minimum angular velocity $\Omega_{\min }$ increases with decreasing compactness of the gravastars. This behavior sets an additional constraint on the parameters of the gravastars that will be subject to the ergoregion instability: less compact gravastars would have to rotate with $\Omega>\Omega_{K}$ to form an ergoregion and are therefore also free from the instability.

Interestingly, there is a non-small portion of the space of parameters $(\mu, \delta)$ where very rapidly rotating gravastars exist, do not possess an ergoregion and are therefore stable. We recall, in fact, that while black holes have their angular momentum bounded by the Kerr limit (i.e. $J / M^{2} \leq 1$ ), stars (and gravastars!) are not subject to this constraint. As a result, as long as they are spinning below the mass-shedding limit, gravastar models can be built that are stable and even have $J / M^{2}>1$. This is shown in Fig. 5, which reports the minimum angular momentum necessary for the existence of an ergoregion, as a function of both $\mu$ and $\delta$. Gravastars with parameters $(\mu, \delta)$ given below a curve labeled with some value of $J_{\min } / M^{2}$ will be stable if rotating with angular momentum smaller than $J_{\min }$ (models on the curve are marginally stable, i.e. with $\tau=\infty$, for that value of $J_{\min }$ ). This is the second important result of this paper: not all ultra-compact astrophysical objects rotating with $J / M^{2} \sim 1$ must be black holes. This conclusion is thus less restrictive than the one drawn by Cardoso et al. in ref. [2]. 


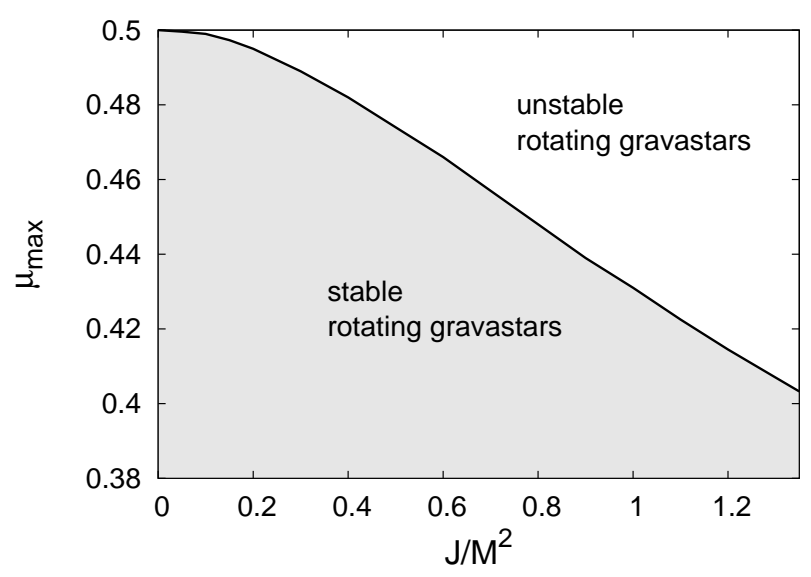

FIG. 6: Maximum compactness $\mu_{\max }$ that a stable gravastar can have in terms of the angular momentum $J / M^{2}$. For a given value of $J$, gravastars with $\mu>\mu_{\max }(J)$ will be unstable. The maximum value for the compactness is obtained for $\delta \rightarrow 0$. This figure should be compared with the corresponding Fig. 1 of ref. [11].

Note that the $\Omega$ is not constant along the $J_{\min }-$ const. lines but, rather, it increases in the direction of the region labeled $\Omega_{\min }\left(J_{\min }\right)>\Omega_{K}$. However, as the angular momentum is increased, the maximum compactness allowed for the stable models is also reduced, thus indicating a new bound on the compactness of stable models. We show this additional constraint on the gravastar's compactness in Fig. 6, which reports the maximum allowed compactness $\mu_{\max }$ as a function of the angular momentum $J / M^{2}$. [This figure should be compared with the corresponding Fig. 1 of ref. [11] where the constraint on the compactness was shown as a function of the thickness for nonrotating gravastars.]

Focusing on $J / M^{2} \simeq 1$, a case which is astrophysically very interesting [20, 21], Fig. 6 shows that it is possible to construct stable rotating gravastars with $J / M^{2} \simeq 1$ as long as their compactness is less than $\mu_{\max } \lesssim 0.43$. Such a compactness is clearly smaller than that of a black hole with the same angular momentum (i.e. $\mu=1$ ), but much larger than the typical compactness for compact stars and neutron stars, i.e. $0.15 \lesssim \mu \lesssim 0.2$. (We recall that the maximum compactness for for perfect fluid nonrotating spheres is given by the Buchdahl-Bondi limit $\mu=4 / 9 \simeq 0.44$ [22, 23]). It is still unclear whether astronomical electromagnetic observations will (ever) be able to distinguish a stable rapidly rotating gravastar with $\mu \sim 0.43$ from a rotating black hole with $J / M^{2} \sim 1$ (see the discussion in ref. [24]). Yet, the constraints emerging from Fig. 6 provide other means (besides the measurement of quasi-normal modes [11]) in which astronomical observations could be used to distinguish (rotating) gravastars from (rotating) black holes.

So far all of the considerations made were on the general properties of rotating gravastars. However, to fix the ideas and also provide some reference numbers on the frequencies and timescales for the ergoregion, we now discuss in more detail one typical case which we will assume to be our reference model ( $c f$. Table \). More specifically, we consider a gravastar with mass $M=10 M_{\odot}$ and inner and outer radii $r_{1}=1.8 M \simeq 27 \mathrm{~km}, r_{2}=2.2 M \simeq 32 \mathrm{~km}$ (or, alternatively, $\mu \simeq 0.45, \delta / M=0.4$ ). Its mass-shedding spin frequency will be $\nu_{K}=\Omega_{K} / 2 \pi \simeq 990 \mathrm{~Hz}$. Such a gravastar will be stable (because without ergoregion), for $J / M^{2} \lesssim 0.13$ (or $\left.\Omega / \Omega_{K} \lesssim 0.10\right)$. Conversely, if the gravastar is set to rotate at a higher rate, namely with $J / M^{2} \simeq 0.22$ (or $\Omega / \Omega_{K} \simeq 0.18$ ), an ergoregion will be present and the instability will develop over a timescale $\tau \sim 10^{17} \mathrm{~s}$, with $\ell=m=4$ being the lowest unstable mode. Note that such a timescale is comparable with the Hubble timescale and thus the rotating gravastar will be physically stable although mathematically unstable (a similar result holds true also for compact uniform-density stars [12]). In practice, it is necessary to spin the same gravastar up to $J / M^{2} \simeq 0.61$ (or $\Omega / \Omega_{K} \simeq 0.50$ ) in order for the instability to develop on a timescale of the order of $1 \mathrm{~s}$, with the $\ell=m=1$ being the lowest unstable mode.

A more complete picture of the real and imaginary parts of the eigenfrequencies for our representative rotating gravastars is shown in Fig. 7 where we have extensively explored the parameter space $(\mu, \delta, J)$. More specifically, the top row shows in the left panel the timescale $\tau$ of the instability (with $\ell=m=1$ ) as a function of the angular momentum $J$ of the gravastar, for fixed thickness of the shell $\delta / M=0.4$ and different compactnesses $\mu$ (from left to right $\mu=0.47,0.45,0.44$ and 0.43 ). As a reference the horizontal line shows a timescale of 1 s for gravastars with $M=10 M_{\odot}$. Similarly, the right panel of the top row gives the frequency $\Sigma$ as a function of $J$ for the same cases in the left panel. The bottom row, on the other hand, shows the same quantities as the top one, but for gravastars with fixed compactness $\mu=0.45$ and varying thickness of the shell $\delta$ (from left to right $\delta / M=0.6,0.5,0.4,0.3$, and 0.2 ).

Note that the timescale for the growth of the instability increases over-exponentially with decreasing angular momentum $J$, and this is why a small spin down of the gravastar is sufficient to make it "effectively stable" even if it possesses an ergoregion and is mathematically unstable. Note also that the frequency $\Sigma$ increases only linearly with decreasing $J$. Finally, to aid those interested in reproducing our results and for code-testing purposes, we have collected in Table $\amalg$ some numerical values for the first unstable mode relative to our reference gravastar ( $c f$. Table \). Clearly, the values of $\tau$ are very sensitive to $J$, but the big difference in $\tau$ seen between the gravastar with $J / M^{2}=0.4$ and the one with $J / M^{2}=0.2$ is also due to the fact that in the first case the lowest unstable mode is still $\ell=m=1$, while in the second case it is already $\ell=m=5$.

The concluding remark of this Section should be one of caution. All of our treatment is based on the slow-rotation approximation, yet we have stretched it to compute also models with $\Omega / \Omega_{K}=1$. Furthermore, while the WKB formulas we used were derived in the high- $m$ limit, we have effectively used them also for very low values of $m$ and found, in particular, that most rotating gravastars are unstable already for $\ell=m=1$ mode, as a result of their high compactness. Al- 

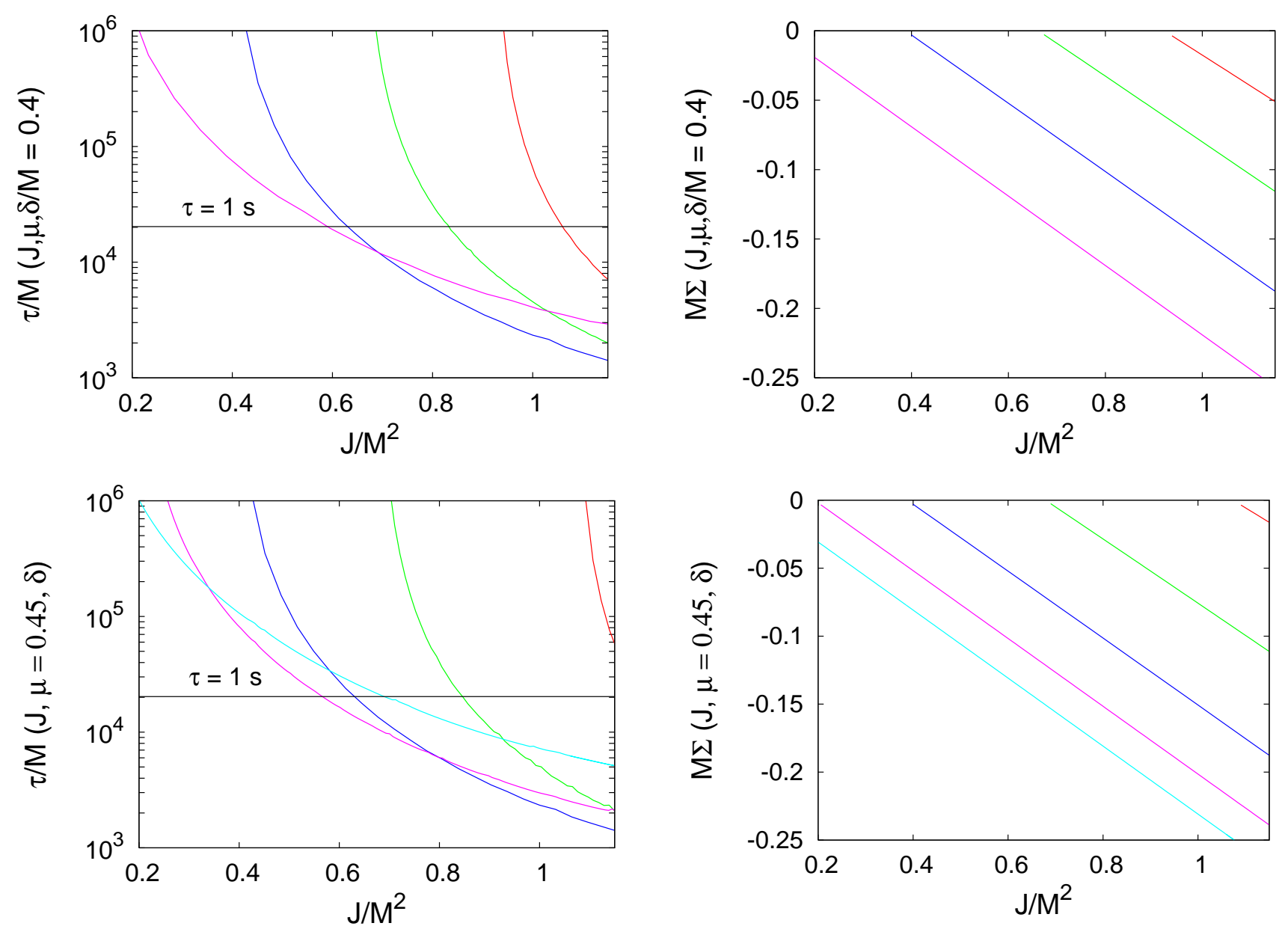

FIG. 7: Top row, left panel: Timescale $\tau$ of the instability (with $\ell=m=1$ ) as a function of the angular momentum $J$ of the gravastar, for fixed thickness of the shell $\delta / M=0.4$ and different compactnesses $\mu$ (from left to right $\mu=0.47,0.45,0.44$ and 0.43 ). The horizontal line shows a timescale of 1 s for gravastars with $M=10 M_{\odot}$. Top row, right panel: Frequency $\Sigma$ of the instability as a function of $J$ for the same cases in the left panel. Bottom row: The same as in the top row, but for gravastars with fixed compactness $\mu=0.45$ and varying thickness of the shell $\delta$ (from left to right $\delta / M=0.6,0.5,0.4,0.3$ and 0.2 ).

though our approach is not dissimilar to the one made in related works [2, 12], it is important to underline that we expect our estimates to be accurate only for slowly rotating gravastars. On the other hand, we also believe that the qualitative (and possibly quantitative) picture derived here will remain unchanged also when a more sophisticated analysis is performed.

\section{CONCLUSIONS}

Motivated by recent work on this subject [2], we have investigated the ergoregion instability in rotating gravastars, exploring a large space of parameters and taking into account the limits on the thickness of the matter shell and on the compactness. While we confirm the results of Cardoso et al. [2] for the models they have considered, we also draw two conclusions which are less restrictive than theirs. Firstly, we find that models of rotating gravastars without an ergoregion (and therefore stable) can be constructed even for extreme rotation
TABLE II: Typical values of $J / M^{2}, r_{a}, r_{b}, r_{c}, \Sigma$ and $\tau$ for for the first unstable mode of the gravastar with $\mu=0.45$ and $\delta / M=0.4$. Note that all values but the last one refer to the $\ell=m=1$ mode.

\begin{tabular}{lllllll}
\hline \hline$J / M^{2}$ & $r_{a} / M$ & $r_{b} / M$ & $r_{c} / M$ & $M \Sigma$ & $\tau / M$ & unstable mode \\
\hline 1.2 & 1.24 & 2.048 & 4.31 & -0.20 & $1.24 \times 10^{3}$ & $\ell=m=1$ \\
1.0 & 1.24 & 2.052 & 5.76 & -0.15 & $2.35 \times 10^{3}$ & $\ell=m=1$ \\
0.8 & 1.24 & 2.056 & 8.88 & -0.10 & $5.96 \times 10^{3}$ & $\ell=m=1$ \\
0.6 & 1.25 & 2.061 & 18.1 & -0.052 & $2.73 \times 10^{4}$ & $\ell=m=1$ \\
0.4 & 1.25 & 2.066 & 350 & -0.0027 & $1.12 \times 10^{7}$ & $\ell=m=1$ \\
0.2 & 1.70 & 1.982 & 592 & -0.0017 & $1.19 \times 10^{30}$ & $\ell=m=5$ \\
\hline \hline
\end{tabular}

rates, namely for models with $J / M^{2} \sim 1$. Hence, not all rotating gravastars possess an ergoregion. Secondly, because stable gravastar models with $J / M^{2} \sim 1$ can be constructed, we conclude that not all ultra-compact astrophysical objects rotating with $J / M^{2} \sim 1$ must be black holes.

Besides clarifying these two important aspects of rotating 
gravastars, our analysis also helps to further constrain the properties of these ultra-compact objects. Building on our initial work [11], in fact, we have computed an additional constraint on the maximum compactness of a gravastar which is to be stable to the ergoregion instability. Such a maximum compactness is still much larger than that of typical neutron stars but also smaller than that of black holes with $J / M^{2} \sim 1$. This should help in the important effort of distinguishing (rotating) gravastars from (rotating) black holes.

\section{Acknowledgments}

It is a pleasure to thank Shin'ichirou Yoshida for numerous discussions and suggestions, and Vitor Cardoso for sharing some of his numerical results and for useful comments. CBMHC gratefully acknowledges the Alexander von Humboldt Society for a postdoctoral fellowship; part of this work was supported in part by the DFG grant SFB/Transregio 7.

\section{APPENDIX}

We review the derivation of the WKB formulas 25 and (26) used in this paper, following closely the treatment given in ref. [12]. We have chosen to include this Appendix in the paper, even though the usual WKB approximation is standard in quantum mechanics textbooks, because the case in question is somewhat more complicated than the usual textbook exercises. The equation to be solved is [cf. eq. (22)]

$$
\psi_{, r r}+m^{2} T(r, \Sigma) \psi=0
$$

which has four different regions with distinct physical behavior, as described in Section III We are interested in finding the purely outgoing modes of this equation. These are given as poles of the scattering amplitude $S \equiv C_{\text {out }} / C_{\text {in }}$, where $C_{\text {out }}$ and $C_{\text {in }}$ are functions of the complex frequency $\sigma(\Sigma=\sigma / m$ and we assume a $e^{i \sigma t}$ dependence for $\psi$ ) and denote the amplitude of the outgoing and incoming waves at infinity, respectively. In a pole of $S$, we will have $C_{\text {in }}=0$ and $C_{\text {out }} \neq 0$. For the modes with small imaginary part, $\Re(\sigma) \gg \Im(\sigma)$ it will be sufficient to determine the eigenvalue $\sigma$ approximately as a real frequency (on the real axis). In this spirit we define an auxiliary function $\bar{S}(\sigma)=\left[S\left(\sigma^{*}\right)\right]^{*}$, such that if $S$ has a pole at $\sigma_{\mathrm{p}}$, then $\bar{S}$ has a pole at $\sigma_{\mathrm{p}}^{*}$, where (*) denotes complex conjugation.

If we restrict ourselves now to $\sigma \in \mathbb{R}$, we will have conservation of energy and

$$
|S|=1 \Rightarrow S(\sigma)[S(\sigma)]^{*}=1 \Rightarrow S(\sigma)=[\bar{S}(\sigma)]^{-1},
$$

where the last result is obtained because $\sigma$ is real. As the relation above is valid for all real values of $\sigma$, it is valid everywhere $S$ and $\bar{S}$ are analytic functions. Therefore, returning to the complex plane, we can state that if $\bar{S}$ has a pole at $\sigma_{\mathrm{p}}^{*}$, then $S$ has a zero at $\sigma_{\mathrm{p}}^{*}$ (and still a pole at $\sigma_{\mathrm{p}}$, which we assume to be simple), and can be written approximately as

$$
S=e^{2 i \delta_{0}} \frac{\sigma-\sigma_{\mathrm{p}}^{*}}{\sigma-\sigma_{\mathrm{p}}} \Rightarrow S=e^{2 i \delta_{0}} \frac{\sigma-\sigma_{\mathrm{r}}-\mathrm{i} / \tau}{\sigma-\sigma_{\mathrm{r}}+\mathrm{i} / \tau}
$$

Now we turn to the specific form of the solutions of eq. A28 in the four different regions as discussed in Sect.III, region I or inner "forbidden" region, region II or "allowed" region, region III or "potential barrier", region IV: $r>r_{c}$ or external "allowed" region ( $c f$. Fig. 2). The connection formulas for the wave function to the left and right of a turning point can be found in many standard textbooks on quantum mechanics (e.g. [25]) and amount to

$$
\begin{aligned}
& \frac{2}{\sqrt{k}} \cos \left(\int_{x}^{a} k d x-\frac{\pi}{4}\right) \rightarrow \frac{1}{\sqrt{\kappa}} \exp \left(-\int_{a}^{x} \kappa d x\right) \\
& \frac{2}{\sqrt{k}} \sin \left(\int_{x}^{a} k d x-\frac{\pi}{4}\right) \leftarrow-\frac{1}{\sqrt{\kappa}} \exp \left(\int_{a}^{x} \kappa d x\right)
\end{aligned}
$$

for a turning point $x=a$ to the right of the classical region, and

$$
\begin{aligned}
& \frac{1}{\sqrt{\kappa}} \exp \left(-\int_{x}^{b} \kappa d x\right) \rightarrow \frac{2}{\sqrt{k}} \cos \left(\int_{b}^{x} k d x-\frac{\pi}{4}\right) \\
& -\frac{1}{\sqrt{\kappa}} \exp \left(\int_{x}^{b} \kappa d x\right) \leftarrow \frac{2}{\sqrt{k}} \sin \left(\int_{b}^{x} k d x-\frac{\pi}{4}\right)
\end{aligned}
$$

for a turning point $x=b$ to the left of the classical region. In this notation, the equation to be solved is $d^{2} \psi / d x^{2}+$ $k^{2}(x) \psi=0$ or $d^{2} \psi / d x^{2}-\kappa^{2}(x) \psi=0$, with $k^{2}, \kappa^{2}>0$. We will need this formulas in what follows, to connect the wave function in the different regions. The form of the radial function $\psi(r)$ in region I is determined by the regularity condition at the center ( $\psi$ must vanish at $r=0$ ),

$$
\psi_{\mathrm{I}}=\frac{C_{1}}{r^{1 / 2}|T|^{1 / 4}} \exp \left(-m \int_{r}^{r_{a}} \sqrt{|T|} d r\right) .
$$

Using now the connection formula A33, we can obtain the form of the radial wave function in region II (connecting through the first turning point $r=r_{a}$ )

$$
\begin{aligned}
\psi_{\mathrm{II}}= & \frac{C_{1} e^{\mathrm{i} \zeta}}{r^{1 / 2} T^{1 / 4}} \exp \left(\mathrm{i} m \int_{r_{b}}^{r} \sqrt{T} d r\right)+ \\
& +\frac{C_{1} e^{-\mathrm{i} \zeta}}{r^{1 / 2} T^{1 / 4}} \exp \left(-\mathrm{i} m \int_{r_{b}}^{r} \sqrt{T} d r\right),
\end{aligned}
$$

where $\zeta \equiv-m \int_{r_{a}}^{r_{b}} \sqrt{|T|} d r-\pi / 4$. We can now write the solution in regions III and IV as

$$
\begin{aligned}
\psi_{\mathrm{III}}= & \frac{C_{2}}{r^{1 / 2}|T|^{1 / 4}} \exp \left(-m \int_{r_{b}}^{r} \sqrt{|T|} d r\right)+ \\
& +\frac{C_{3}}{r^{1 / 2}|T|^{1 / 4}} \exp \left(m \int_{r_{b}}^{r} \sqrt{|T|} d r\right), \\
\psi_{\mathrm{IV}}= & \frac{C_{4}}{r^{1 / 2} T^{1 / 4}} \exp \left(\mathrm{i} m \int_{r_{c}}^{r} \sqrt{T} d r\right)+ \\
& +\frac{C_{5}}{r^{1 / 2} T^{1 / 4}} \exp \left(-\mathrm{i} m \int_{r_{c}}^{r} \sqrt{T} d r\right) .
\end{aligned}
$$


Combining the expressions for $\psi_{\mathrm{II}}, \psi_{\mathrm{III}}$ and $\psi_{\mathrm{IV}}$ with the connection formulas A31-A34, we can relate the amplitudes of the waves before and after crossing the potential barrier. This is done as in a standard scattering exercise, demanding the continuity of the wave function and its first derivative across the turning points $r_{b}$ and $r_{c}$. The result, as stated in ref. [25], is remarkably simple and best expressed in matrix notation:

$$
\left(\begin{array}{c}
C_{1} e^{\mathrm{i} \zeta} \\
C_{1} e^{-\mathrm{i} \zeta}
\end{array}\right)=\frac{1}{2}\left(\begin{array}{cc}
2 \eta+\frac{1}{2 \eta} & \mathrm{i}\left(2 \eta-\frac{1}{2 \eta}\right) \\
-\mathrm{i}\left(2 \eta-\frac{1}{2 \eta}\right) & 2 \eta+\frac{1}{2 \eta}
\end{array}\right)\left(\begin{array}{c}
C_{4} \\
C_{5}
\end{array}\right),
$$

where $\eta \equiv \exp \left(m \int_{r_{b}}^{r_{c}} \sqrt{|T|} d r\right)$. We can now identify the amplitudes $C_{4}$ and $C_{5}$ with the amplitudes $C_{\text {in }}$ and $C_{\text {out }}$ of the incoming and outgoing waves at infinity that we have introduced in the definition of $S$. If $\Sigma$ is negative, the case in which we have unstable modes, then $C_{4}=C_{\text {out }}$ and $C_{5}=C_{\text {in }}$. Inverting now eq. A39 in order to obtain $C_{4}$ and $C_{5}$ in terms of $C_{1}$, they can be substituted in the definition of $S$ to give

$$
S=\frac{C_{4}}{C_{5}}=\frac{\left(4 \eta^{2}+1\right) e^{\mathrm{i} \zeta}-\mathrm{i}\left(4 \eta^{2}-1\right) e^{-\mathrm{i} \zeta}}{\mathrm{i}\left(4 \eta^{2}-1\right) e^{\mathrm{i} \zeta}+\left(4 \eta^{2}+1\right) e^{-\mathrm{i} \zeta}} .
$$

As we have discussed above in the beginning of this Appendix, we are interested in finding the purely outgoing modes, which are poles of the scattering amplitude $S$. Also, we made the assumption that this poles will occur for complex frequencies $\sigma_{\mathrm{p}}$ which lie on the complex plane, but very close to the real axis. But the imaginary part of $\sigma_{\mathrm{p}}$ will only be very small if the "barrier penetration" integral $\eta$ is very large. Taking the limit of $S$ as $\eta \rightarrow \infty$, we have

$$
S \simeq \frac{e^{\mathrm{i} \zeta}-\mathrm{i} e^{-\mathrm{i} \zeta}}{\mathrm{i} e^{\mathrm{i} \zeta}+e^{-\mathrm{i} \zeta}}=-\mathrm{i} \text { for } \eta \rightarrow \infty
$$

unless we have $e^{\mathrm{i} \zeta}-\mathrm{i} e^{-\mathrm{i} \zeta}=0$, in which case we can write $S$ as

$$
S=\mathrm{i} \frac{-4 \eta^{2}\left(\mathrm{i} e^{\mathrm{i} \zeta}+e^{-\mathrm{i} \zeta}\right)-\left(\mathrm{i} e^{\mathrm{i} \zeta}-e^{-\mathrm{i} \zeta}\right)}{4 \eta^{2}\left(\mathrm{i} e^{\mathrm{i} \zeta}+e^{-\mathrm{i} \zeta}\right)-\left(\mathrm{i} e^{\mathrm{i} \zeta}-e^{-\mathrm{i} \zeta}\right)}=\mathrm{i}
$$

Therefore we can see that $S$ will have a resonance at a frequency $\sigma_{n}$ near the frequency for which $e^{\mathrm{i} \zeta}-\mathrm{i} e^{-\mathrm{i} \zeta}=0$ and thus $\zeta=n \pi+\pi / 4$, with $n$ an integer. We can now write $\zeta$ as a series expansion around $\sigma_{n}, \zeta(\sigma) \simeq n \pi+\pi / 4+\alpha_{n}\left(\sigma-\sigma_{n}\right)$, where

$$
\alpha_{n}=\left.\frac{d}{d \sigma}\left(m \int_{r_{a}}^{r_{b}} \sqrt{T} d r\right)\right|_{\sigma=\sigma_{n}} .
$$

Substituting now $\zeta(\sigma)$ into eq. A40, we obtain at first order in $\left(\sigma-\sigma_{n}\right)$ and dropping the index $n$ on $\alpha_{n}$

$$
S=\frac{-\alpha\left(\sigma-\sigma_{n}\right)+1 / 4 \eta^{2}+\mathrm{i}\left[\alpha\left(\sigma-\sigma_{n}\right)+1 / 4 \eta^{2}\right]}{-\alpha\left(\sigma-\sigma_{n}\right)+1 / 4 \eta^{2}-\mathrm{i}\left[\alpha\left(\sigma-\sigma_{n}\right)+1 / 4 \eta^{2}\right]}
$$

which can then be rewritten in the form of eq. A30,

$$
S=\mathrm{i} \frac{\sigma-\sigma_{n}-\mathrm{i} / 4 \eta^{2} \alpha}{\sigma-\sigma_{n}+\mathrm{i} / 4 \eta^{2} \alpha}
$$

Combining the definition of $\zeta$ and its expansion and taking $\sigma=\sigma_{n}$ we finally obtain eq. 25 and comparing eqs. (A30) and (A45, we obtain eq. 26, both presented in Section III.
[1] P. O. Mazur and E. Mottola, Proc.Nat.Acad.Sci. 111, 9545 (2004).

[2] V. Cardoso, P. Pani, M. Cadoni and M. Cavaglià, Phys.Rev.D 77, 124044 (2008).

[3] M. Visser and D. L. Wiltshire, Class. Quant. Grav. 21, 1135 (2004).

[4] B. M. N. Carter, Class. Quant. Grav. 22, 4551 (2005).

[5] N. Bilić , G. B. Tupper and R. D. Viollier, JCAP 0602, 013 (2006).

[6] F. S. N. Lobo, Class. Quant. Grav. 23, 1525 (2006).

[7] F. S. N. Lobo and A. V. B. Arellano, Class. Quant. Grav. 24, 1069 (2007).

[8] D. Horvat, S. Ilijic and A. Marunovic, "Electrically charged gravastar configurations", arXiv:0807.2051 1 1 [gr-qc].

[9] A. E. Broderick and R. Narayan, Class. Quant. Grav. 24, 659 (2007).

[10] A. De Benedictis, D. Horvat, S. Ilijic, S. Kloster and K. S. Viswanathan, Class. Quant. Grav. 23, 2303 (2006).

[11] C. B. M. H. Chirenti and L. Rezzolla, Class. Quant. Grav. 24, 4191 (2007).

[12] N. Comins and B. F. Schutz, Proc. R. Soc. Lond. A364, 211 (1978).
[13] S. Yoshida and Y. Eriguchi, Mon. Not. R. Astron. Soc. 282, 580 (1996).

[14] C. Cattoen, T. Faber and M. Visser, Class. Quant. Grav. 22, 4189 (2005).

[15] M. R. Mbonye and D. Kazanas, Phys. Rev. D 72, 024016 (2005).

[16] J. B. Hartle, Astroph. J. 150, 1005 (1967).

[17] J. M. Bardeen, in Proc. Les Houches, 1972 (Gordon and Breach Science Publisher, New York), p.241.

[18] J. G. Simmonds and J. E. Mann,Jr., A First Look at Perturbation Theory (Dover Publications, New York, 1988), p.71-80.

[19] R. A. Konoplya, Phys. Rev. D68, 024018 (2003).

[20] J. E. McClintock, R. Shafee, R. Narayan, R. A. Remillard, S. W. Davis and L. X. Li, Astrophys. J. 652, 518 (2006).

[21] L. Rezzolla, T. W. Maccarone, S. Yoshida and O. Zanotti, Mon. Not. Roy. Ast. Soc. 344, L37 (2003).

[22] H. A. Buchdahl, Phys. Rev. 116, 1027(1959).

[23] D. Martin and M. Visser, Class. Quant. Grav. 20, 3699 (2003).

[24] M. A. Abramowicz, W. Kluźniak, and J.-P. Lasota, A\&A 396, L31 (2002)

[25] E. Merzbacher, Quantum Mechanics (John Wiley \& Sons, New York, 1961), p.112-134. 